\title{
Uso de probióticos como adición al tratamiento periodontal no quirúrgico en pacientes con periodontitis.
}

\section{Use of probiotics as an adjunctive therapy to non-surgical periodontal treatment in patients with periodontitis.}

\author{
Francisca Salgado ${ }^{1,2}$, Lukas Díaz ${ }^{1,2}$, Valentina Jubal ${ }^{1,2}$, Natacha Oyarzo ${ }^{1,2^{*}}$
}

\author{
1. Escuela de Odontología, Facultad de Medicina, \\ Pontificia Universidad Católica de Chile, Santiago, \\ Chile \\ 2. Proyecto Epistemonikos, Santiago, Chile \\ * Correspondencia Autor: Natacha Oyarzo \\ | Dirección: Centro Evidencia UC, Pontificia \\ Universidad Católica de Chile, Diagonal Paraguay \\ 476, Santiago, Chile | E-mail: noyarzo@uc.cl
}

\begin{abstract}
RESUMEN
Introducción: El tratamiento periodontal no quirúrgico corresponde a la terapia estándar para la periodontitis crónica. En este contexto, se ha propuesto que el uso de probióticos orales como complemento al tratamiento estándar podría mejorar los resultados obtenidos clínicamente, sin embargo no existe claridad respecto a su efectividad. Métodos: Se realizó una búsqueda en Epistemonikos: base de datos de revisiones sistemáticas en salud mantenida mediante el cribado de múltiples fuentes de información, la cuál incluye MEDLINE, EMBASE, Cochrane, entre otras. Se extrajeron los datos desde las revisiones identificadas y un análisis de los datos de los estudios primarios. Se realizó un metanálisis y se preparó una tabla de resumen de los resultados utilizando el método GRADE. Resultados y conclusiones: Se Identificaron seis revisiones sistemáticas que en conjunto incluyeron 14 estudios primarios, todos correspondientes a ensayos aleatorizados. De los resultados analizados se concluye que el uso de probióticos orales como complemento al tratamiento no quirúrgico de periodontitis crónica probablemente resulta en nula o poca diferencia en la reducción de la profundidad de sondaje, el sangrado al sondaje y una ganancia de nivel de inserción clínico en los sitios afectados. Por otro lado, no se encontraron estudios que evaluaran el riesgo de presentar halitosis en pacientes sometidos a terapia periodontal no quirúrgica con adición de probióticos.
\end{abstract}

\section{PALABRAS CLAVE}

Periodontitis; Probióticos; terapia periodontal no quirúrgica; Pulido radicular; Epistemonikos; GRADE.

\begin{abstract}
Introduction: Non-surgical periodontal treatment is the standard treatment to chronic periodontitis. It has been proposed that the use of oral probiotics as an adjunctive therapy may improve clinical outcomes, however there is no clarity regarding its effectiveness. Methods: We searched in Epistemonikos, the largest database of systematic reviews in health, which is maintained by screening multiple information sources, including MEDLINE, EMBASE, Cochrane, among others. We extracted data from the systematic reviews, reanalyzed data of primary studies, conducted a metaanalysis and generated a summary of findings table using the GRADE approach. Results and conclusions: We identified six systematic reviews including 14 studies overall, of which all were randomized trials. We concluded that the addition of probiotics to non-surgical periodontal treatment probably results in little or no difference in probing depth reduction, clinical attachment level gain and reduction in bleeding on probing. No studies were found evaluating halitosis in patients undergoing non-surgical periodontal treatment with the addition of probiotics.
\end{abstract}

\section{KEY WORDS}

Chronic periodontitis; Probiotics; Non surgical therapy; Scaling and root planing; Epistemonikos; GRADE.

Int. J. Inter. Dent Vol. 14(1); 89-94, 2021. 


\section{PROBLEMA}

La periodontitis es una enfermedad multifactorial, la cual afecta los tejidos de soporte del diente, mediante la respuesta inmune del organismo frente a los patógenos presentes. Esto comienza a desarrollarse por disbiosis generada por los microorganismos ${ }^{[1]}$, principalmente bacterias anaerobias, ubicados en los sitios periodontales que pueden provocar finalmente, junto a otros factores involucrados, la pérdida de soporte periodontal. La periodontitis hoy se clasifica de acuerdo a su severidad y grado, de acuerdo a la nueva clasificación ${ }^{[2]}$.

Dentro de las terapias periodontales, el tratamiento no quirúrgico o convencional consiste en la correcta remoción y desinfección de la superficie radicular del diente, mediante el procedimiento de pulido radicular realizado tradicionalmente con ultrasonido y curetas periodontales. El objetivo de este tratamiento es eliminar y desorganizar la biopelícula microbiana, logrando una superficie lisa y pulida para obtener superficies biocompatibles. El efecto de esta acción lleva a una disminución de la profundidad de los sacos, ganancias de inserción clínica, disminución del sangrado al sondaje y obtención de resultados clínicos favorables para mantener la función, salud y estética de los tejidos periodontales ${ }^{[3]}$.

Como complemento a esta terapia se han propuesto distintos tratamientos que podrían mejorar los resultados clínicos enunciados, logrando mayores ganancias de inserción y resultados clínicos. El uso de probióticos es uno de ellos.

Los probióticos actúan mediante la alteración de la proporción de los microorganismos patógenos aumentando la presencia de las bacterias comensales benéficas ${ }^{[4]}$. Lo anterior implica que adicionar probióticos podría alterar las cargas bacterianas en la cavidad oral y sus tejidos gingivales y estos actuarían como un coadyuvante para lograr mejores resultados clínicos y mejores condiciones de salud gingival[5]. Sin embargo, no existe claridad sobre el verdadero rol de los probióticos y su efectividad al ser adicionados a la terapia convencional.

\section{MÉTODOS}

Se realizó una búsqueda en Epistemonikos, la mayor base de datos de revisiones sistemáticas en salud, la cual es mantenida mediante búsquedas en múltiples fuentes de información, incluyendo MEDLINE, EMBASE, Cochrane, entre otras. Se extrajeron los datos desde las revisiones identificadas y se analizó los datos de los estudios primarios. Con esta información, se generó un resumen estructurado denominado FRISBEE (Friendly Summaries of Body of Evidence using Epistemonikos), siguiendo un formato preestablecido, que incluye mensajes clave, un resumen del conjunto de evidencia (presentado como matriz de evidencia en Epistemonikos), metanálisis del total de los estudios cuando sea posible, una tabla de resumen de resultados con el método GRADE y una sección de otras consideraciones para la toma de decisión.

\section{Mensajes clave}

- La adición de probióticos al tratamiento periodontal no quirúrgico probablemente resulta en nula o poca diferencia en la reducción de la profundidad de sondaje, ganancia de nivel de inserción clínica y la reducción de sangrado al sondaje.

- No se encontraron estudios que evaluaran halitosis de pacientes sometidos a terapia periodontal no quirúrgica con adición de probióticos.
Acerca del conjunto de evidencia para esta pregunta

Cuál es la evidencia
Véase matriz de evidencia en
Epistemonikos más abajo.

Encontramos seis revisiones sistemáticas ${ }^{[6,7,8,9,10,11]}$ que en conjunto incluyeron 14 estudios primarios ${ }^{[12,13,14,15 \text {, }}$ $16,17,18,19,20,21,22,23,24,25]$, de los cuales, todos corresponden a ensayos aleatorizados.

Todos los ensayos incluyeron a pacientes con periodontitis crónica sin ningún factor modificante de la enfermedad. No se mencionó profundidad de los sacos ni algún indicador de severidad del diagnóstico

Qué tipo de pacientes incluyeron los estudios* de periodontitis. Se excluyeron pacientes fumadores y pacientes con enfermedades sistémicas como diabetes mellitus.

La edad promedio de los pacientes en los ensayos varió entre los 30 y 50 años. Solo un ensayo no reportó información sobre la edad de sus participantes ${ }^{[23]}$.

Todos los ensayos evaluaron el uso de probióticos como complemento al tratamiento no quirúrgico para periodontitis crónica comparado con el tratamiento no quirúrgico sin complementos adicionales.

La administración de probióticos fue vía oral en forma de tabletas. Se utilizó Lactobacillus Reuteri en diez ensayos ${ }^{[12,13,14,15,17,18,19,20,21,23]}$, Lactobacillus rhamnosus en dos ensayos $^{[22,25]}$, Streptococcus faecalis en un ensayo ${ }^{[24]}$, Clostridium butyricum en un ensayo ${ }^{[24]}$, Bacillus mesentericus en un ensayo ${ }^{[24]}$, Lactobacillus sporogenes en un ensayo ${ }^{[24]}$, Streptococcus salivarius en un ensayo ${ }^{[16]}$, Bifidobacterium animalis en un ensayo ${ }^{[23]}$.

Qué tipo de intervenciones incluyeron los estudios*

El consumo era de 2 tabletas por día por: 2 semanas en dos ensayos [15,17], 3 semanas en tres ensayos ${ }^{[13,20,24]}$, 12 semanas en dos ensayos ${ }^{[19,21]}, 1$ mes en un ensayo ${ }^{[23]}, 3$ meses en dos ensayos ${ }^{[22,25]}, 4$ meses en un ensayo[ ${ }^{[16]}$, sin tiempo especificado en cuatro ensayos ${ }^{[12,14,18,19]}$.

En todos los ensayos, ambos grupos recibieron tratamiento periodontal no quirúrgico,el que consistió en instrucción de higiene oral para los individuos y luego raspado y alisado de las superficies radiculares con curetas y ultrasonido.

Del total de ensayos, dos no utilizaron placebo para el grupo contro|[24,25].

Los ensayos reportaron múltiples desenlaces, los cuales fueron agrupados por las revisiones sistemáticas de la siguiente manera:

-Reducción de profundidad de sondaje (PPD)

-Ganancia de Nivel de inserción clínico (CAL)

Qué tipo de desenlaces -Reducción Sangrado al sondaje (BOP) midieron
•Reducción de patógenos periodontales.

- Índice gingival.

- Índice de placa.

El seguimiento promedio de los ensayos varió desde un mes hasta un año. De los 14 ensayos, dos tuvieron un seguimiento menor a 3 meses $^{[15,18]}$ y uno no reporta la duracion $^{[12]}$.

* La información sobre los estudios primarios es extraída desde las revisiones sistemáticas identificadas, no directamente desde los estudios, a menos que se especifique lo contrario. 


\section{RESUMEN DE LOS RESULTADOS}

La información sobre los efectos de probióticos en adición a terapia periodontal no quirúrgica en pacientes adultos con periodontitis está basada en 7 ensayos, que incluyeron 242 pacientes. El resto de los ensayos no presentaban datos reutilizables para metaanálisis.

Siete ensayos midieron el desenlace de reducción de profundidad de sondaje (242 pacientes) ${ }^{[13,14,15,17,19,20,21]}$. Siete ensayos midieron el desenlace ganancia de nivel de inserción clínica (242 pacientes) $[13,14,15,17,19,20,21]$ y tres ensayos midieron el desenlace sangrado al sondaje $(100 \text { pacientes })^{[13,15,21]}$
El resumen de los resultados es el siguiente:

- La adición de probióticos a tratamiento periodontal no quirúrgico probablemente resulta en nula o poca diferencia en la reducción de profundidad de sondaje (certeza de la evidencia moderada).

- La adición de probióticos a tratamiento periodontal no quirúrgico probablemente resulta en nula o poca diferencia en la ganancia de nivel de inserción clínica (certeza de la evidencia moderada).

- La adición de probióticos a tratamiento periodontal no quirúrgico probablemente resulta en nula o poca diferencia en la reducción de sangrado al sondaje (certeza de la evidencia moderada).

- No se encontraron estudios que evaluaran halitosis de pacientes sometidos a terapia periodontal no quirúrgica con adición de probióticos

\begin{tabular}{|c|c|c|c|c|}
\hline \multicolumn{5}{|c|}{ Adición de probióticos a tratamiento convencional para periodontitis } \\
\hline $\begin{array}{l}\text { Pacientes } \\
\text { Intervención } \\
\text { Comparación }\end{array}$ & \multicolumn{4}{|c|}{$\begin{array}{l}\text { Pacientes adultos con periodontitis } \\
\text { Adición de probióticos a tratamiento periodontal no quirúrgico } \\
\text { Tratamiento periodontal no quirúrgico }\end{array}$} \\
\hline \multirow{3}{*}{ Desenlaces } & \multicolumn{2}{|c|}{ Efecto absoluto* } & \multirow{3}{*}{$\begin{array}{l}\text { Efecto } \\
\text { relativo } \\
\text { (IC } 95 \%)\end{array}$} & \multirow{3}{*}{$\begin{array}{l}\text { Certeza de } \\
\text { la evidencia } \\
\text { (GRADE) }\end{array}$} \\
\hline & $\begin{array}{c}\text { SIN } \\
\text { probióticos } \\
\end{array}$ & $\begin{array}{c}\text { CON } \\
\text { probióticos } \\
\end{array}$ & & \\
\hline & \multicolumn{2}{|c|}{ Diferencia: pacientes por 1000} & & \\
\hline \multirow{2}{*}{$\begin{array}{l}\text { Reducción de } \\
\text { profundidad de } \\
\text { sondaje } \\
\text { (PPD)** }\end{array}$} & 0,36 milímetros & 0,75 milímetros & \multirow[b]{2}{*}{--} & \multirow{2}{*}{$\begin{array}{c}\oplus \oplus \oplus \circ \\
\text { Moderada }^{1}\end{array}$} \\
\hline & \multicolumn{2}{|c|}{$\begin{array}{l}\text { DM:0,39 milímetros más } \\
\text { (Margen de error: } 0,01 \text { a } 0,76 \text { más) }\end{array}$} & & \\
\hline \multirow{2}{*}{$\begin{array}{l}\text { Ganancia de } \\
\text { nivel de } \\
\text { inserción clínica } \\
(\mathrm{CAL}) * * *\end{array}$} & 0,4 milímetros & 0,55 milímetros & \multirow[b]{2}{*}{--} & \multirow[b]{2}{*}{$\begin{array}{c}\oplus \oplus \oplus \circ \\
\text { Moderada }^{1}\end{array}$} \\
\hline & \multicolumn{2}{|c|}{$\begin{array}{c}\text { DM:0,15 milímetros más } \\
\text { (Margen de error: 0,01 puntos menos a 0,31 } \\
\text { más) }\end{array}$} & & \\
\hline \multirow{2}{*}{$\begin{array}{l}\text { Reducción de } \\
\text { sangrado al } \\
\text { sondaje } \\
(\text { BOP)**** }\end{array}$} & $66,8 \%$ & $72,65 \%$ & \multirow[b]{2}{*}{--} & \multirow{2}{*}{$\begin{array}{c}\oplus \oplus \oplus \circ \\
\text { Moderada }^{1}\end{array}$} \\
\hline & \multicolumn{2}{|c|}{$\begin{array}{c}\text { DM: } 5,85 \% \text { más } \\
\text { (Margen de error: } 4,89 \text { a } 6,82 \text { más) }\end{array}$} & & \\
\hline Halitosis & \multicolumn{2}{|c|}{$\begin{array}{c}\text { El desenlace halitosis no fue medido o } \\
\text { reportado. }\end{array}$} & -- & -- \\
\hline
\end{tabular}


*El promedio SIN probióticos está basado en el promedio del grupo control en los estudios/en el promedio del estudio de mayor peso/en la mediana del grupo control de los estudios. El promedio CON probióticos (y su margen de error) está calculado a partir de la diferencia de medias (y su margen de error).

** La profundidad del sondaje corresponde a la medición en milímetros desde el margen gingival a la base del surco periodontal, con una sonda periodontal estandarizada. La reducción de la profundidad de sondaje (PPD) se obtiene al comparar el valor inicial de PPD versus el valor posterior a la terapia periodontal. Se consideró una reducción de $3 \mathrm{~mm}$ como la diferencia mínima importante. El período de seguimiento fue entre 3 semanas hasta 2 años.

*** El nivel de inserción corresponde a la medición en milímetros realizada con una sonda periodontal estandarizada desde el límite amelocementario hasta la base del surco periodontal. Para determinar una ganancia, se debe restar el valor inicial de CAL con el valor posterior a la terapia periodontal. Se consideró $2 \mathrm{~mm}$ como la diferencia mínima importante. El período de seguimiento fue entre 3 semanas hasta 2 años.

**** El sangrado al sondaje corresponde a la proporción de sitios que sangra hasta 15 segundos después del sondaje respecto al número total de sitios. Para obtener el valor porcentual del índice $\mathrm{BOP}$, se divide el número de sitios que sangraron en el total de sitios en boca y se multiplica por 100 . Se consideró un valor $\leq 10 \%$ como la diferencia mínima importante. El período de seguimiento fue entre 3 semanas hasta 2 años.

${ }^{1}$ Se disminuyó un nivel de certeza de evidencia por inconsistencia, dado que los distintos estudios llegaron a conclusiones diferentes (I2>79\%).

Siga el enlace para acceder a la versión interactiva de esta tabla (Interactive Summary of Findings - iSoF)

\section{Acerca de la certeza de la evidencia (GRADE)*} $\oplus \oplus \oplus \oplus$

Alta: La investigación entrega una muy buena indicación del efecto probable. La probabilidad de que el efecto sea sustancialmente distinto ${ }^{\dagger}$ es baja.

\section{$\oplus \oplus \oplus \bigcirc$}

Moderada: La investigación entrega una buena indicación del efecto probable. La probabilidad de que el efecto sea sustancialmente distinto ${ }^{\dagger}$ es moderada.

\section{$\oplus \oplus \bigcirc \bigcirc$}

Baja: La investigación entrega alguna indicación del efecto probable. Sin embargo, la probabilidad de que el efecto sea sustancialmente distinto† es alta.

\section{$\oplus \mathrm{OOO}$}

Muy baja: La investigación no entrega una estimación confiable del efecto probable. La probabilidad de que el efecto sea sustancialmente distinto† es muy alta.

*Esto es también denominado 'calidad de la evidencia' o 'confianza en los estimadores del efecto'. tSustancialmente distinto = una diferencia suficientemente grande como para afectar la decisión 


\section{OTRAS CONSIDERACIONES PARA LA TOMA DE DECISIÓN}

\section{A quién se aplica y a quién no se aplica esta evidencia}

- Los resultados de este resumen son aplicables a todos los pacientes adultos sanos con periodontitis.

- Esta evidencia no aplicaría para pacientes fumadores, diabéticos, pacientes en etapa de terapia periodontal de soporte ni a pacientes que reciban antibióticos como terapia adjunta.

\section{Sobre los desenlaces incluidos en este resumen}

- Los desenlaces incluidos en este resumen son considerados críticos para la toma de decisiones clínicas, de acuerdo a la opinión de los autores de este resumen, coincidiendo con lo evaluado por las revisiones sistemáticas incluidas.

- El desenlace halitosis es considerado crítico tanto para los expertos clínicos como para el paciente. Sin embargo, no fue reportado en ninguna de las revisiones sistemáticas identificadas.

\section{Balance daño/beneficio y certeza de la evidencia}

- De acuerdo a la evidencia incluida en este resumen, los resultados muestran que probablemente no hay un beneficio clínicamente relevante al adicionar probióticos a la terapia periodontal no quirúrgica en relación a la reducción de la profundidad de sondaje, ganancia de inserción clínica y sangrado al sondaje.

- Por otro lado, tampoco se ha reportado que la adición de probióticos genere un aumento en el riesgo de eventos adversos u otro posible daño para la salud oral y periodontal.

- Considerando lo anterior, el balance daño/beneficio probablemente está a favor de no adicionar probióticos a la terapia periodontal no quirúrgica.

\section{Consideraciones de recursos}

- Ninguna de las revisiones sistemáticas mencionan los costos asociados al uso de probióticos. Sin embargo, la intervención no debiese elevar de manera importante el costo del tratamiento.

\section{Qué piensan los pacientes y sus tratantes}

- Enfrentados a la evidencia disponible, la mayoría de los pacientes y tratantes deberían inclinarse en contra de la utilización de probióticos como terapia adjunta al tratamiento no quirúrgico.

\section{Diferencias entre este resumen y otras fuentes}

- Cinco ${ }^{[6,7,8,9,11]}$ de las seis revisiones sistemáticas identificadas consideran que la evidencia actual es insuficiente para obtener conclusiones con respecto a esta intervención.

- Una de las revisiones ${ }^{[10]}$ concluyó que la adición de probióticos mostraba un efecto clínico significativo. Esto podría deberse a que este efecto fue reportado solamente a corto plazo.

- Ninguna de las guías clínicas identificadas recomiendan el uso de probióticos en estos pacientes con periodontitis crónica ${ }^{[26,27]}$

\section{¿Puede que cambie esta información en el futuro?}

- La probabilidad de que las conclusiones de este resumen cambien por futura evidencia es moderada, debido a la certeza de la evidencia que presentan los outcomes.

- No identificamos ninguna revisión sistemática o ensayos clínicos aleatorizados en curso en International prospective register of systematics reviews (PROSPERO) del National Institute for Health Research ni en el International Clinical Trials Registry Platform de la Organización Mundial de la Salud.

\section{CÓMO REALIZAMOS ESTE RESUMEN}

Mediante métodos automatizados y colaborativos recopilamos toda la evidencia relevante para la pregunta de interés y la presentamos en una matriz de evidencia.

Siga el enlace para acceder a la versión interactiva: Adición de probióticos a tratamiento convencional para periodontitis

\section{NOTAS}

Si con posterioridad a la publicación de este resumen se publican nuevas revisiones sistemáticas sobre este tema, en la parte superior de la matriz se mostrará un aviso de "nueva evidencia".

Este artículo es parte del proyecto síntesis de evidencia de Epistemonikos. Se elabora con una metodología preestablecida, siguiendo rigurosos estándares metodológicos y proceso de revisión por pares interno. Cada uno de estos artículos corresponde a un resumen, denominado FRISBEE (Friendly Summary of Body of Evidence using Epistemonikos), cuyo principal objetivo es sintetizar el conjunto de evidencia de una pregunta específica, en un formato amigable a los profesionales clínicos. Sus principales recursos se basan en la matriz de evidencia de Epistemonikos y análisis de resultados usando metodología GRADE. Mayores detalles de los métodos para elaborar este FRISBEE están descritos aquí (http://dx.doi.org/10.5867/medwave.2014.06.5997)

La Fundación Epistemonikos es una organización que busca acercar la información a quienes toman decisiones en salud, mediante el uso de tecnologías. Su principal desarrollo es la base de datos Epistemonikos (www.epistemonikos.org).

\section{DECLARACIÓN DE CONFLICTOS DE INTERESES}

Los autores declaran no tener conflictos de intereses con la materia de este artículo.

\section{AGRADECIMIENTOS}

Este resumen de evidencia fue elaborado con el apoyo metodológico del Centro Evidencia UC, Facultad de Medicina, Pontificia Universidad Católica de Chile. 


\section{Bibliografía}

1. Lang NP, Bartold PM. Periodontal health. J Periodontol. 2018 Jun;89 Suppl 1:S9S16. doi: 10.1002/JPER.16-0517. PMID: 29926938

2. Caton JG, Armitage G, Berglundh T, Chapple ILC, Jepsen S, Kornman KS, et al. A new classification scheme for periodontal and peri-implant diseases and conditions - Introduction and key changes from the 1999 classification. J Clin Periodontol. 2018 Jun;45 Suppl 20:S1-S8. doi: 10.1111/jcpe.12935. PMID: 29926489.

3. Dentino A, Lee S, Mailhot J, Hefti AF. Principles of periodontology. Periodontol 2000. 2013 Feb;61(1):16-53. doi: 10.1111/j.1600-0757.2011.00397.x. PMID: 23240942.

4. Darveau RP, Hajishengallis G, Curtis MA. Porphyromonas gingivalis as a potential community activist for disease. J Dent Res. 2012 Sep;91(9):816-20. doi: 10.1177/0022034512453589. PMID: 22772362; PMCID: PMC3420389.

5. Chimenos-Küstner E, Giovannoni ML, Schemel-Suárez M. Dysbiosis as a determinant factor of systemic and oral pathology: importance of microbiome. Med Clin (Barc). 2017 Oct 11;149(7):305-9. doi: 10.1016/j.medcli.2017.05.036. PMID: 28669517.

6. Martin-Cabezas R, Davideau JL, Tenenbaum H, Huck O. Clinical efficacy of probiotics as an adjunctive therapy to non-surgical periodontal treatment of chronic periodontitis: a systematic review and meta-analysis. J Clin Periodontol. 2016 Jun:43(6):520-30. doi: 10.1111/jcpe.12545. PMID: 26970230.

7. Ikram S, Hassan N, Raffat MA, Mirza S, Akram Z. Systematic review and meta-analysis of double-blind, placebo-controlled, randomized clinical trials using probiotics in chronic periodontitis. J Investig Clin Dent. 2018 Aug;9(3):e12338. doi 10.1111/jicd.12338. PMID: 29604177.

8. Ho SN, Acharya A, Sidharthan S, Li KY, Leung WK, McGrath C, et al. A systematic review and meta-analysis of clinical, immunological, and microbiological shift in periodontitis after nonsurgical periodontal therapy with adjunctive use of probiotics. $J$ Evid Based Dent Pract. 2020 Mar;20(1):101397. doi: 10.1016/j.jebdp.2020.101397. PMID: 32381406

9. Vives-Soler A, Chimenos-Küstner E. Effect of probiotics as a complement to nonsurgical periodontal therapy in chronic periodontitis: a systematic review. Med Oral Patol Oral Cir Bucal. 2020 Mar 1;25(2):e161-e167. doi: 10.4317/medoral.23147. PMID: 31893476; PMCID: PMC7103449.

10. Song D, Liu XR. Role of probiotics containing Lactobacillus reuteri in adjunct to scaling and root planing for management of patients with chronic periodontitis: a meta-analysis. Eur Rev Med Pharmacol Sci. 2020 Apr;24(8):4495-505. doi: 10.26355/eurrev_202004_21032. PMID: 32373987.

11. Donos N, Calciolari E, Brusselaers N, Goldoni M, Bostanci N, Belibasakis GN. The adjunctive use of host modulators in non-surgical periodontal therapy. A systematic review of randomized, placebo-controlled clinical studies. J Clin Periodontol. 2020 Jul;47 Suppl 22:199-238. doi: 10.1111/jcpe.13232. PMID: 31834951.

12. Szkaradkiewicz AK, Stopa J, Karpiński TM. Effect of oral administration involving a probiotic strain of Lactobacillus reuteri on pro-inflammatory cytokine response in patients with chronic periodontitis. Arch Immunol Ther Exp (Warsz). 2014 Dec;62(6):495-500. doi: 10.1007/s00005-014-0277-y. Epub 2014 Feb 9. PMID: 24509697: PMCID: PMC4244533.

13. Tekce M, Ince G, Gursoy H, Dirikan Ipci S, Cakar G, Kadir T, et al. Clinical and microbiological effects of probiotic lozenges in the treatment of chronic periodontitis: a 1-year follow-up study. J Clin Periodontol. 2015 Apr;42(4):363-72. doi: 10.1111/ jcpe.12387. Epub 2015 Apr 10. PMID: 25728888

14. Pelekos G, Ho SN, Acharya A, Leung WK, McGrath C. A double-blind, paralleledarm, placebo-controlled and randomized clinical trial of the effectiveness of probiotics as an adjunct in periodontal care. J Clin Periodontol. 2019 Dec;46(12):1217-27. doi: 10.1111/jcpe.13191. Epub 2019 Oct 28. PMID: 31479530

15. Vivekananda MR, Vandana KL, Bhat KG. Effect of the probiotic Lactobacilli reuteri (Prodentis) in the management of periodontal disease: a preliminary randomized clinical trial. J Oral Microbiol. 2010 Nov 2;2. doi: 10.3402/jom.v2i0.5344. PMID: 21523225; PMCID: PMC3084569.

16. Mani A, Saini R, Saini SR. Efficacy of oral probiotics as an adjunct to scaling and root planing in nonsurgical treatment outcome of Generalized chronic periodontitis patients: a Clinico-microbiological study. Int J Exp Dent Sci. 2017;6(1):6-13. Doi: 10.5005/JP-JOURNALS-10029-1145

17. Penala S, Kalakonda B, Pathakota KR, Jayakumar A, Koppolu P, Lakshmi BV, et al. Efficacy of local use of probiotics as an adjunct to scaling and root planing in chronic periodontitis and halitosis: A randomized controlled trial. J Res Pharm Pract. 2016 Apr-Jun;5(2):86-93. doi: 10.4103/2279-042X.179568. PMID: 27162801; PMCID: PMC4843589.

18. Costacurta M, Sicuro L, Margiotta S, Ingrasciotta I, Docimo R. Clinical effects of lactobacillus reuteri probiotic in treatment of chronic periodontitis. A randomized controlled trial. Oral \& Implantology. 2018 Oct-Dec;1(4):191-8.

19. Laleman I, Yilmaz E, Ozcelik O, Haytac C, Pauwels M, Herrero ER, et al. The effect of a streptococci containing probiotic in periodontal therapy: a randomized controlled trial. J Clin Periodontol. 2015 Nov:42(11):1032-41. doi: 10.1111/ icpe.12464. PMID: 26427036.

20. İnce G, Gürsoy H, İpçi ŞD, Cakar G, Emekli-Alturfan E, Yılmaz S. Clinical and biochemical evaluation of lozenges containing lactobacillus reuteri as an adjunct to non-surgical periodontal therapy in chronic periodontitis. J Periodontol. 2015 Jun;86(6):746-54. doi: 10.1902/jop.2015.140612. PMID: 25741580.

21. Teughels W, Durukan A, Ozcelik O, Pauwels M, Quirynen M, Haytac MC. Clinical and microbiological effects of Lactobacillus reuteri probiotics in the treatment of chronic periodontitis: a randomized placebo-controlled study. J Clin Periodontol. 2013 Nov;40(11):1025-35. doi: 10.1111/jcpe.12155. PMID: 24164569; PMCID: PMC3908359.

22. Morales A, Carvajal P, Silva N, Hernandez M, Godoy C, Rodriguez G, et al. Clinical effects of Lactobacillus rhamnosus in non-surgical treatment of chronic periodontitis: a randomized placebo-controlled trial with 1-year follow-up. J Periodontol. 2016 Aug;87(8):944-52. doi: 10.1902/jop.2016.150665. PMID: 26944407.

23. Invernici MM Salvador SL, Silva PHF Soares MSM, Casarin R, Palioto DB, et al. Effects of Bifidobacterium probiotic on the treatment of chronic periodontitis: A randomized clinical trial. J Clin Periodontol. 2018 Oct;45(10):1198-210. doi: 10.1111/ jcpe.12995. PMID: 30076613; PMCID: PMC6221043.

24. Dhaliwal PK, Grover V, Malhotra R, Kapoor A. Clinical and microbiological investigation of the effects of probiotics combined with scaling and root planing in the management of chronic periodontitis: a randomized, controlled study. J Int Acad Periodontol. 2017 Jul 1:19(3):101-108. PMID: 31473697.

25. Morales A, Gandolfo A, Bravo J, Carvajal P, Silva N, Godoy C, et al. Microbiological and clinical effects of probiotics and antibiotics on nonsurgical treatment of chronic periodontitis: a randomized placebo- controlled trial with 9-month follow-up. J App Oral Sci. 2018 Jan 18:26:e20170075. doi: 10.1590/1678-7757-2017-0075. PMID: 29364340; PMCID: PMC5777419.

26. Secretaría de Salud Pública. Orientaciones técnicas para la prevención y tratamiento de las enfermedades gingivales y periodontales. MINSAL: Chile, 2017 27. Cobb CM. Clinical significance of non-surgical periodontal therapy: an evidencebased perspective of scaling and root planing. J Clin Periodontol. 2002 May;29 Suppl 2:6-16. PMID: 12010523. 